\title{
NONCOMMUTATIVE LOCALIZATION
}

\author{
BY JOACHIM LAMBEK
}

0 . Introduction. To motivate this exposition, let us begin by looking at an example. If $\boldsymbol{Z}$ is the ring of integers and $p$ is a prime number, we have embeddings $\boldsymbol{Z} \rightarrow \boldsymbol{Z}_{p} \rightarrow \hat{\boldsymbol{Z}}_{p}$. Here the first arrow indicates "localization at $p ", \boldsymbol{Z}_{p}$ being the local ring of quotients at $p$. We may take this to be the ring of rationals with denominators prime to $p$, described explicitly as a direct limit

$$
\boldsymbol{Z}_{\boldsymbol{p}}=\underline{\lim }\{\operatorname{Hom}((n), \boldsymbol{Z}) \mid p \nmid n\}
$$

where $(n)=n \boldsymbol{Z}$ is the principal ideal generated by $n, n$ running over all positive integers not divisible by $p$. The second arrow indicates " $p$-adic completion". $\hat{\boldsymbol{Z}}_{p}$ is the completion of $\boldsymbol{Z}_{p}$ (incidentally also of $\boldsymbol{Z}$ ) in the $p$-adic topology. It is also known as the ring of $p$-adic integers and may be described explicitly as an inverse limit

$$
\hat{\boldsymbol{Z}}_{p}=\lim _{\{}\left\{\boldsymbol{Z}_{p} /\left(p^{k}\right) \mid k \geqq 0\right\},
$$

where $\left(p^{k}\right)=p^{k} \boldsymbol{Z}_{p}$ is the principal ideal generated by $p^{k}, k$ running over all natural numbers.

In commutative algebra one is always told first to localize and then to complete. It is therefore not surprising that the combination of these two processes can be described in a simpler fashion than either. In fact, $\hat{z}_{p}$ is the ring of endomorphisms of the Prüfer group

$$
\boldsymbol{Z} /\left(p^{\infty}\right)=\underline{\lim }\left\{\boldsymbol{Z} /\left(p^{k}\right) \mid k \geqq 0\right\} .
$$

The latter may also be regarded as the group of all rationals with denominators powers of $p$ modulo $\boldsymbol{Z}$. More significantly, it is the injective hull of $\boldsymbol{Z} /(p)$.

We want to generalize this result in two directions. First, instead of $\boldsymbol{Z}$

An expanded version of an invited address delivered to the 696th meeting of the American Mathematical Society at Dartmouth College, Hanover, New Hampshire, on September 1, 1972. An earlier version Localisation et complétion is to have appeared in "Séminaire d'algèbre non commutative 1971-1972, publications mathématiques d'Orsay". \$7 describes joint work with Gerhard Michler, and $\S \S 8$ and 9 report on a collaboration with Basil Rattray; received by the editors January 8, 1973.

AMS (MOS) subject classifications (1970). Primary 16A08; Secondary 16A52.

Key words and phrases. Localization, torsion theory, ring of quotients, injective module, $I$-adic topology, prime ideal, bicommutator, density theorem, Ore condition, Artin-Rees property. 
we wish to consider an arbitrary ring $R$, assumed to be associative with unity element. When $R$ is commutative Noetherian, this was done by Matlis [M2]. Secondly, instead of localizing at a prime, we wish to discuss a more general process of localization.

With the prime number $p$ there are associated several other objects:

(1) the prime ideal $(p)=p Z$,

(2) the multiplicative set $\boldsymbol{Z}-(p)$,

(3) the filter of ideals $\{(n) \mid p \nmid n\}$,

(4) the injective module $Z /\left(p^{\infty}\right)$.

Localization at prime ideals and, more generally, at multiplicative sets is a well-known classical process for commutative rings. Localization at certain filters of right ideals has been considered by Bourbaki [B4] and Gabriel [G1], while localization at injective modules was introduced by Findlay and the present author in a special situation in [F2], but the same arguments work in general [L2]. The last two methods are equivalent; also equivalent is the method of torsion radicals due to Gabriel [G1], Maranda [M1] and Goldman [G3]. Moreover all these results can be expressed in the language of (hereditary) torsion theories, studied by Dickson [D2] and the present author [L1], [L2]. The literature abounds in methods of localization that are equivalent to the above, e.g. Chew [C1], or are special cases of the above, e.g. Silver [S3]. In at least one case, the localization of Cohn [C2], a comparison still escapes us.

1. Localization at injectives. We shall describe localization at an injective. Let $I$ be a given injective right $R$-module. A right $R$-module $A$ is called torsion (with respect to $I$ ) if $\operatorname{Hom}_{R}(A, I)=0 ; A$ is called torsion free if $A$ is isomorphic to a submodule of $I^{\alpha}$ for some cardinal $\alpha$; and $A$ is called divisible if $I(A) / A$ is torsion free. Here, and throughout this exposition, $I(A)$ denotes the injective hull of $A$.

Some people are wary of injectives, because they tend to be huge and may be difficult to construct. They can be avoided; in fact, all information about the injective $I$ is contained in any essential submodule of $I$, no matter how small. Thus, let $I=I(M)$ be the injective hull of $M$, then $A$ is torsion (with respect to $I$ ) if and only if

$$
\forall_{a \in A} \forall_{0 \neq m \in M} \exists_{r \in R} a r=0 \quad \& \quad m r \neq 0 .
$$

Again, $A$ is divisible (with respect to $I$ ) if and only if, for all right ideals $D$ of $R$ such that $R / D$ is torsion and for all $f \in \operatorname{Hom}_{R}(D, A)$,

$$
\exists_{a \in A} \forall_{d \in D} f(d)=a d .
$$

The reader will appreciate that injectives are useful for stating such definitions more concisely, and we shall retain them for this reason. 
It is easily seen that every module $A$ has a unique submodule $T(A)$ such that $T(A)$ is torsion and $A / T(A)$ is torsion free:

$$
T(A)=\left\{a \in A \mid \operatorname{Hom}_{R}(a R, I)=0\right\} .
$$

$T(A)$ is called the torsion submodule of $A$. Putting $T(I(A) / A)=D(A) / A$, where $A \subseteq D(A) \subseteq I(A)$, we obtain the divisible hull $D(A)$ of $A$. $D$ is not a functor, but its restriction to torsion free modules is. Writing $Q(A)=$ $D(A / T(A)$ ), we do obtain a functor $Q$, the localization functor (with respect to $I)$. In fact, the inclusion \{torsion free modules $\rightarrow$ Mod $R$ has the left adjoint $A \mapsto A / T(A)$ and the inclusion \{torsion free divisible modules\} $\rightarrow$ \{torsion free modules\} has the left adjoint $B \mapsto D(B)$, hence the inclusion \{torsion free divisible modules $\rightarrow \rightarrow$ Mod $R$ has the left adjoint $A \mapsto Q(A)$.

The reflector $A \mapsto Q(A)$ is exact. However, we prefer to regard $Q$ as a functor from $\operatorname{Mod} R$ into $\operatorname{Mod} R$. As such it is left exact, but not in general right exact.

$Q(A)$ is also called the module of quotients of $A$ (with respect to $I$ ). $Q(R)$ is a ring, the ring of right quotients of $R$. The canonical module homomorphism $R \rightarrow Q(R)$ is a ring homomorphism, every torsion free divisible $R$-module is a $Q(R)$-module, and every $R$-homomorphism between torsion free divisible modules is a $Q(R)$-homomorphism.

We hasten to give two illustrations in the case $R=Z$. When $I=Q$, the words "torsion", "torsion free" and "divisible" have their usual meaning. When $I=\mathbf{Z} /\left(p^{\infty}\right)$, they mean " $p$-torsion", " $p$-torsion free" and " $p$-divisible". A $Z$-module is here called $p$-torsion if every element has order prime to $p$; it is called $p$-divisible if every element is divisible by every integer prime to $p$.

2. Localization at filters and multiplicative sets. With any injective $I$ we shall associate the filter of right ideals

$$
\mathscr{D}_{I}=\left\{D \leqq_{r} R \mid \operatorname{Hom}_{R}(R / D, I)=0\right\} .
$$

This is an "idempotent" filter in the sense of [B4] or [G1]. Conversely, given any idempotent filter $\mathscr{D}$, one may form the injective

$$
I_{\mathscr{D}}=\Pi\left\{I(R / K) \mid K \leqq_{r} R \quad \& \quad \forall_{r \notin K^{-1}} r^{-1} \notin \mathscr{D}\right\},
$$

where $r^{-1} K=\{s \in R \mid r s \in K\}$. One easily shows that $\mathscr{D}_{I_{\mathscr{g}}}=\mathscr{D}$, but $I_{\mathscr{D}_{I}}$ is only "similar" to $I$, two injectives being called similar if they give rise to the same localization functor $Q$, that is, if each is isomorphic to a submodule of a power of the other.

Suppose $\mathscr{D}$ corresponds to $I$. Writing

$$
L(A)=\lim _{\longrightarrow}\left\{\operatorname{Hom}_{R}(D, A) \mid D \in \mathscr{D}\right\}
$$


and

$$
T(A)=\left\{a \in A \mid a^{-1} 0 \in \mathscr{D}\right\},
$$

one can show that $Q(A)=L(A / T(A))=L(L(A))$.

When $\Sigma$ is a multiplicative subset of $R$ one may introduce the idempotent filter

$$
\mathscr{D}_{\Sigma}=\left\{D \leqq_{\pi} R \mid \forall_{r \in R} r^{-1} D \cap \Sigma \neq \varnothing\right\}
$$

and put $I_{\Sigma}=I_{\mathscr{D}_{\Sigma}}$. The definition of $\mathscr{D}_{\Sigma}$ simplifies to

$$
\mathscr{D}_{\Sigma}=\left\{D \leqq_{r} R \mid D \cap \Sigma \neq \varnothing\right\}
$$

if and only if $R$ satisfies the right Ore condition with respect to $\Sigma$, that is,

$$
\forall_{r \in \boldsymbol{R}} \forall_{\sigma \in \Sigma} \exists_{\boldsymbol{r}^{\prime} \in \boldsymbol{R}^{\prime}} \exists_{\sigma^{\prime} \in \Sigma} r \sigma^{\prime}=\sigma r^{\prime} .
$$

Suppose $h: R \rightarrow R_{\Sigma}$ is any ring homomorphism. One calls the pair $\left(R_{\Sigma}, h\right)$ a classical ring of right quotients of $R$ with respect to $\Sigma$ provided

(a) $\forall_{r \in R} h(r)=0 \Rightarrow \exists_{\sigma \in \Sigma} r \sigma=0$,

(b) $\forall_{\sigma \in \Sigma} h(\sigma)$ is invertible,

(c) $\forall_{q \in R_{\Sigma}} \exists_{r \in R} \exists_{\sigma \in \Sigma} q h(\sigma)=h(r)$.

As Gabriel has shown [G1, Proposition 5, p. 415], $R$ possesses a classical ring of right quotients with respect to $\Sigma$ if and only if $(*)$ and

$$
\forall_{r \in R}\left(\exists_{\sigma \in \Sigma} \sigma r=0 \Rightarrow \exists_{\sigma^{\prime} \in \Sigma} r \sigma^{\prime}=0\right) .
$$

It is clear that $R_{\Sigma}=Q(R)$, the ring of quotients with respect to $I_{\Sigma}$.

The argument in [L5, Proposition 5.5] can be used to show that $(*) \Rightarrow(* *)$ when $R$ is right Noetherian.

Indeed, suppose $\sigma r=0$. Pick the natural number $n$ such that the right annihilator of $\sigma^{n}$ is maximal. Now, by $\left({ }^{*}\right)$, there exists $r^{\prime} \in R$ and $\sigma^{\prime} \in \Sigma$ such that $\sigma^{n} r^{\prime}=r \sigma^{\prime}$. Therefore $\sigma^{n+1} r^{\prime}=\sigma r \sigma^{\prime}=0$. But the right annihilator of $\sigma^{n+1}$ contains that of $\sigma^{n}$, hence coincides with the latter by choice of $n$. Therefore $r \sigma^{\prime}=\sigma^{n} r^{\prime}=0$, and so (**) holds.

In the situation described above, it is easily shown that $R_{\Sigma}$ is flat as a left $R$-module. In general, a left $R$-module $F$ is flat if and only if the right $R$-module $F^{*}=\operatorname{Hom}_{Z}(F, Q / Z)$, called the "character module of $F$ ", is injective.

One may ask when the localization functor $Q$ can be obtained from an injective of the form $F^{*}$ where $F$ is flat. In that case a right module $A$ will be torsion if and only if $A \otimes_{R} F=0$, and a right ideal $D$ of $R$ belongs to the associated idempotent filter if and only if $D F=F$.

This situation arises, for example, when $Q$ is obtained from an injective of the form $I_{\Sigma}$, where $\Sigma$ is assumed to satisfy $\left({ }^{*}\right)$ and $\left({ }^{* *}\right) . Q$ might then also have been obtained from $R_{\Sigma}^{*}$, hence $I_{\Sigma}$ and $R_{\Sigma}^{*}$ are similar.

Another example is $Q \cong \mathrm{id}$, the identity functor on $\operatorname{Mod} R$. In that case 
$Q$ may be obtained from $F^{*}$, where $F$ is a free left $R$-module.

Schelter and Roberts [S2] have shown that every injective is similar to the character module of a flat module if $R$ is commutative Noetherian or if the filter $\mathscr{D}_{I}$ has countable base. On the other hand, when $R$ is the ring of continuous real valued functions defined on the unit interval, they proved that the injective hull of the right $R$-module $R$ is not similar to the character module of a flat module. It follows that, in this last example, the localization functor $Q$ cannot be obtained from a multiplicative set $\Sigma$ satisfying $(*)$ and $(* *)$.

3. Exactness of localization. We are interested in finding examples when the localization functor $Q$ regarded as an endofunctor of $\operatorname{Mod} R$ is exact and not only left exact. It is easily seen that the following conditions are equivalent:

(a) the functor $Q$ is exact,

(b) the class of torsion free divisible modules is closed under cokernels,

(c) every torsion free factor module of a torsion free divisible module is divisible,

(d) $I(A) / A$ is divisible for all torsion free divisible modules $A$.

Other equivalent conditions have been found by Goldman [G3], Hudry [H2], Djabali [D1, Theorem 2.4], Goodearl [G4, Theorem 1.22] and Beachy [B3]. These conditions are clearly satisfied when $R$ is right hereditary.

It has been shown [L2, Proposition 2.4] that every divisible module (with respect to $I$ ) is injective if and only if $I$ has zero singular submodule, that is, for every $0 \neq i \in I, i^{-1} 0$ is not an essential right ideal of $R$. When this is the case, then surely $I(A) / A=0$ for every divisible module $A$, hence $Q$ is exact by condition $(d)$ above.

We may also ask when $Q$ preserves all co-limits. It is well-known that the following conditions are equivalent:

(a) $Q$ preserves all co-limits,

(b) the class of torsion free divisible modules is closed under co-limits,

(c) every $Q(R)$-module is torsion free,

(d) $Q \cong(-) \otimes_{R} Q(R)$,

(e) for every right ideal $D$ in the filter $\mathscr{D}, D Q(R)=Q(R)$.

The last condition is due to Walkers [W1]. Clearly, it is satisfied when $Q(R)=R_{\Sigma}$. Thus, when $R$ is right Noetherian and satisfies the right Ore condition with respect to $\Sigma$, the localization functor $Q$ obtained from $I_{\Sigma}$ preserves all co-limits.

We have now seen many examples in which $Q$ is exact. An example in which $Q$ is not exact has been constructed by Michler [M5].

There are several reasons why one wants to know that $Q$ is exact. Our interest arises from $\S 6$ below, but one can also show that, when $Q$ is exact, 
then $Q(A) \cong A \otimes_{R} Q(R)$ for every finitely presented module $A$.

4. The $I$-adic topology. Given an injective right $R$-module $I$, we may associate with it a topology on every right $R$-module $A$, the so-called $I$-adic topology. This is defined by saying that a fundamental system of open neighborhoods of 0 consists of all possible kernels of homomorphisms $A \rightarrow I^{n}, n$ being any natural number. One easily verifies that $A$ then becomes a topological group, $R$ a topological ring, and $A$ a topological $R$-module. Moreover every $R$-homomorphism is continuous in the $I$-adic topology.

Since $I$ is injective, it is a trivial observation that, when $A$ is endowed with the $I$-adic topology, the induced topology on any submodule $B$ of $A$ is also $I$-adic. Such a statement does not hold in general for the classical $P$-adic topology associated with a given prime ideal $P$ of $R$. A fundamental system of open neighborhoods of 0 in the $P$-adic topology of $A$ consists of all submodules of the form $A P^{n}$, where $n$ is any natural number.

Proposition 4.1. Let $R$ be a commutative Noetherian local ring with maximal ideal $P, A$ a finitely generated right $R$-module, then the I-adic and $P$-adic topologies on $A$ coincide.

For a generalization to the noncommutative case, see $\S 7$ below. One deduces the classical result of Artin-Rees:

COROLlary 4.2. Under the above assumptions on $R$ and $A$, if $A$ is endowed with the P-adic topology, the induced topology on any submodule of $A$ is also P-adic.

If $A$ is endowed with the $I$-adic topology, we may make it Hausdorff by dividing it by the closure of 0 , namely by

$$
\overline{0}=\bigcap\left\{\operatorname{Ker} f \mid \exists_{n \in N} f \in \operatorname{Hom}_{R}\left(A, I^{n}\right)\right\} .
$$

The $I$-adic completion $\hat{A}$ of $A$ is defined as the completion of $A / \overline{0}$ in the $I$-adic topology. One easily sees that

$$
\hat{A}=\lim _{\longleftarrow}\left\{\operatorname{Im} f \mid \exists_{n \in N} f \in \operatorname{Hom}_{R}\left(A, I^{n}\right)\right\} .
$$

The reader is warned that $\hat{A}$ has the inverse limit topology, which is not in general $I$-adic. We note that $\hat{R}$ is a topological ring and that $\hat{A}$ is a topological $\hat{R}$-module.

The problem posed in $\$ 0$ may now be stated thus: find a simple description of $(Q(A))$.

5. The double dual of $A$. Let $I$ be a given injective right $R$-module, $E$ its ring of endomorphisms. We may regard $I$ as a left $E$-right $R$-bimodule. Consider the functors 


$$
\operatorname{Mod} R \stackrel{\operatorname{Hom}_{R}(-, I)}{\longrightarrow}(E \operatorname{Mod})^{\mathrm{op}} \stackrel{\operatorname{Hom}_{E}(-, I)}{\longrightarrow} \operatorname{Mod} R,
$$

the former being the left adjoint of the latter. We are interested in the composition $S$ of these functors, thus

$$
S(A)=\operatorname{Hom}_{E}\left(\left(\operatorname{Hom}_{R}(A, I), I\right)\right) .
$$

$S(A)$ is a kind of double dual of $A$.

The adjunction $\eta(A): A \rightarrow S(A)$ is defined by $\eta(A)(a)(f)=f(a)$, for all $a \in A$ and $f \in \operatorname{Hom}_{R}(A, I)$. We note that $S(R)$ is a ring, the bicommutator $\operatorname{Hom}_{E}(I, I)$ of $I$, and that $\eta(R): R \rightarrow S(R)$ is a ring homomorphism. We write $\eta_{1}(A): A \rightarrow Q(A)$ for the canonical homomorphism of $\S 1$.

Proposition 5.1. (1) $\operatorname{Ker} \eta(A)=T(A)$,

(2) $S(A)$ is torsion free and divisible,

(3) there exists a unique homomorphism $\kappa(A): Q(A) \rightarrow S(A)$ such that $\kappa(A) \eta_{1}(A)=\eta(A)$

(4) $\kappa(A)$ is a monomorphism,

(5) $\kappa(R)$ is a ring homomorphism.

In view of this result, we may write $Q(A) \subseteq S(A)$ and regard $Q(A)$ as a submodule of $S(A), Q(R)$ as a subring of $S(R)$.

Proposition 5.2. $Q(A)=S(A)$ if either of the following equivalent conditions holds

(a) $\operatorname{Hom}_{R}(A, I)$ is finitely generated as a left E-module,

(b) $T(A)$ is open in the I-adic topology of $A$.

COROLlaRY 5.3. $Q(R)$ is the bicommutator of I if either of the following equivalent conditions holds:

(a) I is finitely generated as a left E-module,

(b) $T(R)$ is open in the I-adic topology of $R$.

Condition $(a)$ holds, for example, when $I$ is principal as a left $E$-module, e.g., when $I=I(R)$, and condition $(b)$ is easily checked when $A$ is Artinian. See [M6] and [L2].

If we endow $S(A)$ with the $I$-adic topology, $Q(A)$ becomes a closed submodule. More interesting and more natural is the finite topology on $S(A)$, that is, the topology induced by the product topology of $I^{\operatorname{Hom}_{R}(A, I)}$, $I$ being taken as discrete.

With each $f \in \operatorname{Hom}_{R}(A, I)$ one associates the projection $p(f): S(A) \rightarrow I$ such that $\forall_{s \in S(A)} p(f)(s)=s(f)$. Note that this implies that $p(f) \eta(A)=f$. Then a fundamental system of open neighborhoods of 0 on $S(A)$ is given by $p\left(f_{1}\right)^{-1}(0) \cap \cdots \cap p\left(f_{n}\right)^{-1}(0)=\left\{s \in S(A) \mid s\left(f_{1}\right)=0 \& \cdots \& s\left(f_{n}\right)=0\right\}$, where $f_{1}, \ldots, f_{n} \in \operatorname{Hom}_{R}(A, I)$. This may be put in the more concise form 
Ker $f^{*}$, where $f=\left(f_{1}, \ldots, f_{n}\right): A \rightarrow I^{n}$ and $f^{*}: S(A) \rightarrow I^{n}$ is the canonical "extension" such that $f^{*} \eta(A)=f$. Explicitly

$$
f^{*}(s)=\left(s\left(f_{1}\right), \ldots, s\left(f_{n}\right)\right)=\sum_{i=1}^{n} k_{i} s p_{i}(f),
$$

where the $k_{i}: I \rightarrow I^{n}$ are the canonical injections and the $p_{i}: I^{n} \rightarrow I$ the canonical projections.

Proposition 5.4. The finite topology on $S(A)$ induces the I-adic topology on $Q(A)$. Moreover, $S(A)$ is Hausdorff and complete in the finite topology.

This result provides the motivation for studying the $I$-adic topology at least on torsion free divisible modules. It also raises the question whether $Q(A)$ is dense in $S(A)$.

6. The density theorem. The assumption in the following theorem holds for each $R$-module $A$ if and only if $Q$ is exact, hence for all the examples considered in $\S 3$.

THEOREM 6.1. Assume that every torsion free factor module of $Q(A)$ is divisible. Then

(1) $Q(A)$ is a dense submodule of $S(A)$ in the finite topology,

(2) $S(A)$ endowed with the finite topology is the completion of $Q(A)$ endowed with the I-adic topology,

(3) $S(A)=\lim _{\longleftarrow}\left\{\operatorname{Im} g \mid \exists_{n \in N} g \in \operatorname{Hom}_{R}\left(Q(A), I^{n}\right)\right\}$.

Proof. (2) and (3) are easy consequences of (1). A proof of (1) was given in [L4] using triples. We shall here present a more elementary proof.

Let $s$ be a given element of $S(A)$. A fundamental open neighborhood of $s$ has the form $\{s\}+\operatorname{Ker} f^{*}$, where $f^{*}: S(A) \rightarrow I^{n}$ is the canonical "extension" of $f: A \rightarrow I^{n}$. We claim that this neighborhood meets $Q(A)$, that is, that $f^{*}(s)=f^{*}(q)$ for some $q \in Q(A)$. Thus we want to prove that $f^{*} S(A) \subseteq f^{*} Q(A)$, that is, that $f^{*} S(A) / f^{*} Q(A)=0$. This will follow if we show that it is both torsion and torsion free.

(a) To prove that $f^{*} S(A) / f^{*} Q(A)$ is a torsion module, we take any $g: f^{*} S(A) \rightarrow I$ such that $g f^{*} Q(A)=0$ and try to show that $g=0$. Extend $g$ to $h: I^{n} \rightarrow I$ and calculate, for any $s \in S(A)$,

$$
g f^{*}(s)=h \sum_{i=1}^{n} k_{i} s p_{i}(f)=s\left(\sum_{i=1}^{n} h k_{i} p_{i}(f)\right),
$$

since $s$ is an $E$-homomorphism and $h k_{i} \in E$. Moreover, since $\sum_{i=1}^{n} k_{i} p_{i}=1$, we obtain $g f *(s)=s(h f)=s(0)=0$, because 


$$
h f=h f^{*} \kappa(A) \eta_{1}(A)=g f^{*} \kappa(A) \eta_{1}(A)=0 .
$$

Therefore $g=0$, as required.

(b) To prove that $f^{*} S(A) / f^{*} Q(A)$ is torsion free, we note that $f^{*} Q(A)$ is a factor module of the divisible module $Q(A)$ and that it is torsion free, because it is a submodule of $I^{n}$. By assumption, $f^{*} Q(A)$ is divisible. But $f^{*} S(A) \subseteq I^{n}$ is torsion free, and every factor module of a torsion free module by a divisible module is torsion free [L2, Proposition 0.6], hence $f^{*} S(A) / f^{*} Q(A)$ is torsion free, as was to be shown.

COROllary 6.2. Assume that every torsion free factor module of $Q(R)$ is divisible. Then the I-adic completion of $Q(R)$ is the bicommutator of I. If $R$ is commutative, it is the center of $E$.

REMARK 6.3. Matlis [M2] obtained this result when $R$ is commutative Noetherian and $I=I(R / P), P$ being a prime ideal. In that case he even showed that $E$ is commutative.

We give three examples when $R=Z$.

EXAMPLE 6.4. $I=\boldsymbol{Z} /\left(p^{\infty}\right), p$ prime, $Q(R)=\boldsymbol{Z}_{p}$, the $I$-adic topology on $Q(R)$ is the $p$-adic topology, and $(Q(R))^{\wedge}=\hat{Z}_{p}$ is the ring of $p$-adic integers.

EXAMPLE 6.5. $I=\boldsymbol{Q}, Q(R)=\boldsymbol{Q}$, the $I$-adic topology on $Q(R)$ is discrete, and $(Q(R))^{\wedge}=\boldsymbol{Q}$.

EXAMPLE 6.6. $I=\boldsymbol{Q} / \boldsymbol{Z}, Q(R)=\boldsymbol{Z}$, the $I$-adic topology on $Q(R)$ has as open sets all arithmetic progressions, and $(Q(R))^{\wedge}=\prod_{p \text { prime }} \hat{\mathbf{Z}}_{p}$.

7. Localization at a semiprime ideal. We now return to the question how to localize at a prime ideal or, more generally, at a semiprime ideal $N$ of $R$. On the one hand, one may consider the injective module $I(R / N)$, on the other hand, one may try to make use of the multiplicative set

$$
\mathscr{C}(N)=\left\{r \in R \mid \forall_{s \notin N} r s \notin N\right\}
$$

proposed by Goldie. When $R$ is commutative and $N$ is prime, $\mathscr{C}(N)$ is the complement of $N$. When $R$ is noncommutative and right Noetherian, $\mathscr{C}(N)$ consists of those elements of $R$ which are regular modulo $N$.

The results of this section were obtained jointly with Gerhard Michler.

Proposition 7.1. Let $N$ be a semiprime ideal in the right Noetherian ring $R$. Then one obtains the same localization functor $Q_{N}$ from the injective module $I(R / N)$ as from the multiplicative set $\mathscr{C}(N)$.

Remark 7.2. When $N=P$ is a prime ideal, let $A$ be maximal among right ideals of $R$ not meeting $\mathscr{C}(P)$. Then $I_{P}=I(R / A)$ is an indecomposable injective, it is determined by $P$ uniquely up to isomorphism, and $I(R / P)=I_{P}^{n}$, where $n$ is the right Goldie dimension of the right Noetherian ring $R / N$. 
In the following we shall write $R_{N}=Q_{N}(R)$ and $\tilde{N}$ for the "closure" of $N$ in $R_{N}$ with respect to $I(R / N)$, that is,

$$
\tilde{N}=\left\{q \in R_{N} \mid \operatorname{Hom}_{R}\left(R / q^{-1} h(R), I(R / N)\right)=0\right\} .
$$

THEOREM 7.3. Let $N$ be a semiprime ideal in the right Noetherian ring $R$. Then the following statements are equivalent:

(1) $R$ satisfies the right Ore condition with respect to $\mathscr{C}(N)$,

(2) $\tilde{N}$ is the Jacobson radical of $R_{N}$ and $R_{N} / \tilde{N}$ is a semisimple Artinian ring,

(3) $\tilde{N}$ is a two-sided ideal of $R_{N}$ and $Q_{N}$ is exact,

(4) for all $c \in \mathscr{C}(N), N / c N$ is torsion with respect to $I(R / N)$.

REMARK 7.4. In view of (4), the right Ore condition may be checked by looking only at elements of $N$. Actually, a much smaller ideal may do. Suppose $K$ is an ideal of $R$ contained in $N$ such that $\mathscr{C}(N) \subseteq \mathscr{C}(K)$ and $N^{n} \subseteq K$ for some natural number $n$. Then the above statements are also equivalent to the following:

(5) for all $c \in \mathscr{C}(N), K / c K$ is torsion with respect to $I(R / N)$.

In particular, taking $N$ to be the prime radical of $R$, we can deduce Small's famous theorem which asserts that $R$ has a classical Artinian ring of right quotients provided all elements of $\mathscr{C}(N)$ are regular.

Lemma 7.5. Let $R$ be a right Noetherian ring, $N$ an ideal such that $R / N$ is semisimple Artinian. Then the following statements are equivalent:

(1) for each right ideal $A$ of $R, \exists_{n \in N} A \cap N^{n} \subseteq A N$;

(2) for each element $i \in I(R / N), \exists_{n \in N} i N^{n}=0$;

(3) on every finitely generated right $R$-module, the $N$-adic topology coincides with the $I(R / N)$-adic topology.

Moreover, these statements hold together with the assertion that $N$ is the Jacobson radical of $R$ if and only if

(4) every right ideal of $R$ is closed in the $N$-adic topology.

When $(R, N)$ satisfies (4) or the equivalent statements of Lemma 7.5, we call it a classical right semilocal ring.

Associated with a semiprime ideal $N$ of a right Noetherian ring $R$ there are two closure operations: $\rho$ for right ideals and $\lambda$ for left ideals. For any right ideal $A$ and any left ideal $B$ of $R$ we put

$$
\begin{aligned}
& \rho A=\left\{r \in R \mid \operatorname{Hom}_{R}\left(R / r^{-1} A, I(R / N)\right)=0\right\}, \\
& \lambda B=\text { smallest left ideal } B^{\prime} \text { such that } B \subseteq B^{\prime} \text { and } \mathscr{C}(N) \subseteq \mathscr{C}\left(B^{\prime}\right) .
\end{aligned}
$$

When $A$ is an ideal, then so are $\rho A$ and $\lambda A$, and $\lambda \rho \lambda A=\rho \lambda A$.

THEOREM 7.6. Let $N$ be a semiprime ideal in the right Noetherian ring $R$. Then the following statements are equivalent:

(1) for each right ideal $A$ of $R, \exists_{n \in N} A \cap \lambda N^{n} \subseteq \rho(A N)$; 
(2) for each $i \in I(R / N), \exists_{n \in N} i \lambda N^{n}=0$;

(3) $\tilde{N}$ is an ideal and $\left(R_{N}, \tilde{N}\right)$ is a classical right semilocal ring.

Moreover, these equivalent statements imply that $R$ satisfies the right Ore condition with respect to $\mathscr{C}(N)$.

Statement (1) asserts that $N$ has the symbolic right Artin-Rees property.

REMARK 7.7. Let $N=P$ be a prime ideal in the right Noetherian ring $R$. Then Michler has shown that the equivalent conditions of Theorem 7.6 imply the following:

(4) $R_{N}$ is an $n$ by $n$ matrix ring over a complete local ring $\hat{D}$ with finitely generated Jacobson radical.

The equivalent statements of Theorem 7.6 are satisfied with $N=P$ in the following examples.

Example 7.8 (Matlis). $R$ commutative Noetherian, $P$ a prime ideal.

EXAMPLE 7.9. $R$ right and left Noetherian hereditary prime, $P$ a prime ideal such that $P^{2} \neq P$. In particular (Kuzmanovich) $R$ Noetherian Dedekind prime, $P$ any nonzero prime ideal. In this example $\hat{D}$ is a complete rank one valuation ring.

EXAMPLE 7.10 (MCCONNELL). $R$ the enveloping algebra of a finitely generated nilpotent Lie algebra, $P$ any nonzero prime ideal.

EXAMPLE 7.11 (MichleR). $R=A G$ the group ring of a finite group $G$ with coefficient ring $A, A$ being right Noetherian prime of characteristic zero, $P$ the augmentation ideal.

8. Categorical treatment of localization. In view of the above examples, one has the feeling that the process of localization at a prime ideal $P$ described here is useful when $R$ satisfies the right Ore condition with respect to $\mathscr{C}(P)$, but there is no evidence that this is so when the Ore condition fails. Perhaps one should work in another category than Mod $R$, for instance, the category of $R$ - $R$-bimodules. Before returning to this question, we shall present a categorical treatment of localization that applies to all complete categories, not just to $\operatorname{Mod} R$.

The work described in this section and in $\$ 9$ was done jointly with Basil Rattray.

From now on $\mathscr{A}$ will be any complete category, that is, a category equipped with products and equalizers. Let $I$ be a given object of $\mathscr{A}$. We shall consider the limit closure $L(I)$ of $I$, that is, the smallest full subcategory of $\mathscr{A}$ which contains $I$ and is closed under all limits, that is, under products and equalizers.

The category $L(I)$ is complete and has a co-generator, namely $I$, and the inclusion into $\mathscr{A}$ preserves limits. One might therefore construct a left adjoint to the inclusion functor by means of Freyd's special adjoint functor theorem, provided one knew that the class of subobjects of any object was a set. We shall use a different method for constructing this adjoint, following an idea of Fakir [F1]. 
We write $(A, B)$ for the set of all morphisms $A \rightarrow B$ in $\mathscr{A}$, Ens for the category of sets, and consider the two functors

$$
\mathscr{A} \stackrel{(-, I)}{\longrightarrow} \mathrm{Ens}^{\mathrm{op}} \stackrel{I^{(-)}}{\longrightarrow} \mathscr{A} .
$$

Clearly the former is left adjoint to the latter with adjunction $\eta:$ id $\rightarrow S=I^{(-, I)}$, where $p(f) \eta(A)=f, p(f): S(A) \rightarrow I$ being the canonical projection corresponding to $f \in(A, I)$.

Since $\eta$ is a natural transformation, $\eta(A)$ equalizes the two morphisms $S \eta(A), \eta S(A): S(A) \rightrightarrows S^{2}(A)$. Let $\kappa(A): Q(A) \rightarrow S(A)$ be their equalizer, then there exists a unique morphism $\eta_{1}(A): A \rightarrow Q(A)$ such that $\kappa(A) \eta_{1}(A)=\eta(A)$. Fakir observes that $\left(Q, \eta_{1}\right)$ gives rise to an idempotent triple if and only if $S(\kappa(A))$ is a monomorphism for all $A$.

The following lemma is useful in proving results about $Q$.

LEMma 8.1. $\kappa(A)$ is the joint equalizer of all pairs of maps $S(A) \rightrightarrows I$ which are equalized by $\eta(A): A \rightarrow S(A)$.

We shall call I injective if $(-, I)$ preserves all regular monomorphisms, that is, monos which happen to be equalizers. This notion seems more useful than the corresponding one for arbitrary monomorphisms. We shall call $I \kappa$-injective if $(\kappa(A), I)$ is a mono in $\mathrm{Ens}^{\mathrm{op}}$, that is, a surjection in Ens, for all objects $A$ of $\mathscr{A}$. This amounts to requiring that every morphism $f: Q(A) \rightarrow I$ can be extended to some $f^{\prime}: S(A) \rightarrow I$ such that $f^{\prime} \kappa(A)=f$.

We write Fix $Q$ for the full subcategory of $\mathscr{A}$ consisting of all objects $A$ for which $\eta_{1}(A)$ is an isomorphism. We note that $I$ belongs to Fix $Q$.

THEOREM 8.2. The following statements are equivalent:

(1) I is $\kappa$-injective;

(2) $\left(Q, \eta_{1}\right)$ determines an idempotent triple;

(3) Fix $Q$ is a reflective subcategory of $\mathscr{A}$, the reflector being induced by $Q$;

(4) Fix $Q$ is the limit closure of $I$.

We point out that, according to Fakir, $\left(Q, \eta_{1}, \mu_{1}\right)$ is the "best coapproximation" of the triple $(S, \eta, \mu)$ by an idempotent triple. (The reader will be able to supply $\mu$ and $\mu_{1}$.)

For some purposes, it is more convenient to look at the dual category $\mathscr{B}=\mathscr{A}^{\mathrm{op}}$, which is thus assumed to be co-complete. With any object $P$ of $\mathscr{B}$ we associate a functor $S: \mathscr{B} \rightarrow \mathscr{B}$, which is defined on objects as a co-product of copies of $P: S(B)=\sum_{f \in(P, B)} P . S$ comes equipped with a natural transformation $\varepsilon: S \rightarrow$ id, and we let $\kappa: S \rightarrow Q$ be the co-equalizer of $\varepsilon S, S \varepsilon: S^{2} \rightrightarrows S$. Theorem 8.2 now takes the dual form: 
THEOREM 8.2.* The following statements are equivalent:

(1) $P$ is $\kappa$-projective;

(2) $\left(Q_{1}, \varepsilon_{1}\right)$ determines an idempotent co-triple;

(3) Fix $Q$ is a co-reflective subcategory of $\mathscr{B}$, the co-reflector being induced by $Q$;

(4) Fix $Q$ is the co-limit closure of $P$.

We can interpret the "co-localization" functor $Q$ associated with a projective $P$ in terms of the Eilenberg-Moore category of a triple. The forgetful functor $U=(P,-): \mathscr{B} \rightarrow$ Ens has a left adjoint $F$ with adjunction $\eta$ :id $\rightarrow U F$, and the triple $(U F, \eta, U \varepsilon F)$ on Ens gives rise to the Eilenberg-Moore category Ens ${ }^{U F}$. Let $K: \mathscr{B} \rightarrow \mathrm{Ens}^{U F}$ be the so-called comparison functor and $M$ its left adjoint, then $M K=Q$.

This observation allows one to formulate Linton's condition for $(\mathscr{B}, U)$ to be equational (triplable over Ens), that is, for $K$ to be canonically isomorphic to the identity, in terms of properties of $P$. While such a formulation may not be new, in the present context it immediately leads to applications that appear not to have been noticed before. For example, if $\mathscr{A}$ is any Grothendieck category or if $\mathscr{A}$ is the category of all set-valued sheaves on a small category equipped with a Grothendieck topology, then $\mathscr{A}^{\mathrm{op}}$ is equational.

9. Localization in various categories. The reader will not be surprised to learn of the following illustration of Theorem 8.2.

EXAmple 9.1. $\mathscr{A}=\operatorname{Mod} R, I$ any injective module, $L(I)$ the class of torsion free divisible modules, $Q$ the localization functor constructed in $\S 1$.

The next example is suggested by Delale [D1], who used an idea by $\mathbf{M}$. Artin.

EXAMPLE 9.2. Let $\mathscr{A}$ be the full co-reflective subcategory of the category of all left $R$-right- $R$-bimodules which are generated by their centers. Thus $A$ is in $\mathscr{A}$ if and only if every element $a \in A$ has the form $a=\sum_{i=1}^{n} a_{i} r_{i}$ where $a_{i} r=r a_{i}$ for all $r \in R$. Delale has shown that all injectives in $\mathscr{A}$ are obtained from injective $R$ - $R$-bimodules $I$ by restricting to $C(I)$, the part generated by the center of $I$. Suppose we have computed the functor $S$ corresponding to $I$ in the category of $R$ - $R$-bimodules, then the functor $S_{C}$ corresponding to $C(I)$ in $\mathscr{A}$ is given by $A \mapsto C S(A)$. The associated idempotent functor $Q_{C}$ has the property that $Q_{C}(R)$ is a ring in many cases.

Another method for obtaining two-sided rings of quotients has been discussed by Schelter [S1].

EXAMPle 9.3 . Let $\mathscr{A}=(\operatorname{Mod} R)^{\text {op }}, P$ a finitely generated projective right $R$-module. One computes $Q(A)=\operatorname{Hom}_{R}(P, A) \otimes_{E} P$, where $E=$ $\operatorname{End}_{R}(P)$ is the ring of endomorphisms of $P$. 
EXAMPLE 9.4. Let $\mathscr{A}=$ Ens $^{\mathscr{O} \text { op }}$ be the category of presheaves on a small category $\mathscr{X}$, which may be equipped with a Grothendieck topology $j$. One can construct a huge injective $I$ depending on $j$ such that the associated localization functor $Q$ assigns to each presheaf $A$ the corresponding sheaf $Q(A) . L(I)$ is of course the category of sheaves.

One can do an analogous thing in any elementary topos $\mathscr{A}$ in the sense of Lawvere and Tierney [L9]. Since $\mathscr{A}$ need not be complete, the above construction of $S$ does not work, instead we put $S(A)=I^{\left(I^{A}\right)}$. For $I$ we choose the retract of the subobject classifier $\Omega$ determined by the Heyting endomorphism $j$ of $\Omega$ (Grothendieck topology). Not surprisingly, it turns out that $Q(A)$ will again be the sheaf associated with $A$. However, it happens that Fix $Q$ is not the limit closure of $I$, it must also be closed under internal powers.

EXAMPLE 9.5 . Let $\mathscr{A}$ be the category of uniform spaces, $I$ the closed interval $[0,1]$. Then $I$ is injective and $Q(A)$ is the Samuel compactification of $A$.

EXAMPLE 9.6. Let $\mathscr{A}$ be the category of topological spaces, $I$ again the closed unit interval. Then $I$ is not injective but $\kappa$-injective, by Tietze's theorem, and $Q(A)=\beta(A)$ is the Stone-Čech compactification of $A$. This is essentially the way Cech originally constructed it, except that he defined $Q(A)$ as a closure, not as an equalizer.

EXAMPLE 9.7 . Let $\mathscr{A}$ be the category of partially ordered sets and inf preserving mappings. Then Ballinger [B1] has shown that the partially ordered set 2 is not injective but $\kappa$-injective, and that Fix $Q$ consists of all complete partially ordered sets. He has also investigated other categories of partially ordered sets, semilattices and lattices.

\section{REFERENCES}

The following list of references is by no means complete. Bigger lists will be found in [L2] and [S5].

The reader who is interested in further details is invited to consult the following references:

$\begin{array}{ll}\text { For } \S 1 & \text { see }[\mathbf{L 2}] \\ \text { For } \S 2 & \text { see }[\mathbf{G 1}] \\ \text { For } \S \S 3-6 & \text { see }[\mathbf{L 3}, \mathbf{L 4}] \\ \text { For } \S 7 & \text { see }[\mathbf{L 5}, \mathbf{L 6}, \mathbf{L 7}] \\ \text { For } \S \S 8,9 & \text { see }[\mathbf{L 8}] .\end{array}$

[B1] B. T. Ballinger, Completions of ordered sets, Thesis, McGill University, (to be submitted).

[B2] J. A. Beachy, A characterization of torsionfree modules over rings of quotients, Proc. Amer. Math. Soc. 34 (1972), 15-19.

[B3] - On quotient functors which preserve small projectives (to appear).

[B4] N. Bourbaki, Eléments de mathématique. Fasc. XXVII. Algèbre commutative. Chap. 1: Modules plats. Chap. 2: Localisation, Actualités Sci. Indust., no. 1290, Hermann, Paris, 1961. MR 36 \# 146. 
[C1] K. L. Chew, Closure operations in the study of rings of quotients, Bull. Math. Soc. Nanyang Univ. 1965, 1-20. MR 34 \#4298.

[C2] P. M. Cohn,. Skew fields of fractions, and the prime spectrum of a general ring, Lectures on rings and modules, Lecture Notes in Math., vol. 246, Springer-Verlag, Berlin, 1972, pp. 1-71.

[D1] J. P. Delale, Théorie de la localisation, Thesis, University of Paris (to be submitted).

[D2] S. E. Dickson, A torsion theory for Abelian categories, Trans. Amer. Math. Soc. 121 (1966), 223-235. MR 33 \#162.

[D3] M. Djabali, Anneaux de fractions généralisés, Séminaire d'algèbre non commutative 1971-1972, Publications mathématiques d'Orsay, 17.1-17.12.

[F1] S. Fakir, Monade idempotente associée à une monade, C.R. Acad. Sci. Paris Sér, A-B 270 (1970), A99--A101. MR 41 \# 1828.

[F2] G. D. Findlay and J. Lambek, A generalized ring of quotients. I, II, Canad. Math. Bull. 1 (1958), 77-85, 155-167. MR 20 \#888.

[G1] P. Gabriel, Des catégories abéliennes, Bull. Soc. Math. France 90 (1962), 323-448. MR 38 \# 1144.

[G2] A. W. Goldie, A note on non-commutative localization, J. Algebra 8 (1968), 41-44. MR 36 \# 6442.

[G3] O. Goldman, Rings and modules of quotients, J. Algebra 13 (1969), 10-47. MR 39 \#6914.

[G4] K. R. Goodearl, Torsion for modules, Thesis, University of Washington, Seattle, Wash., 1971.

[H1] A. G. Heinicke, On the ring of quotients at a prime ideal of a right Noetherian ring, Canad. J. Math. 24 (1972), 703-712.

[H2] A. Hudry, Sur la localisation dans une catégorie de modules, C.R. Acad. Sci. Paris Sér. A-B 270 (1970), A925-A928. MR 41 \#6895.

[K1] J. Kuzmanovich, Completions of Dedekind prime rings and second endomorphism rings, Pacific J. Math. 36 (1971), 721-729. MR 44 \# 1696.

[L1] J. Lambek, Completions of categories, Seminar lectures given in 1966 in Zürich, Lecture Notes in Math., no. 24, Springer-Verlag, Berlin and New York, 1966. MR 35 \# 228.

[L2] - Torsion theories, additive semantics, and rings of quotients, Lecture Notes in Math., vol. 177, Springer-Verlag, Berlin and New York, 1971. MR 44 \# 1685

[L3] , Bicommutators of nice injectives, J. Algebra 21 (1972), 60-73.

[L4] - Localization and completion, J. Pure Appl. Algebra 2 (1972), 343-370.

[L5] J. Lambek and G. Michler, The torsion theory at a prime ideal of a right Noetherian ring, J. Algebra 25 (1973), 364-389.

[L6] - Completions and classical localizations of right Noetherian rings, Pacific J.

Math. (to appear).

[L7] - Localization of right Noetherian rings at semiprime ideals (to appear).

[L8] J. Lambek and B. Rattray, Localization at injectives in complete categories, Proc. Amer. Math. Soc. (to appear).

[L9] F. W. Lawvere, Introduction. Toposes, algebraic geometry and logic, Lecture Notes in Math., vol. 274, Springer-Verlag, Berlin and New York, 1972, pp. 1-12.

[L10] L. Lesieur, La torsion associée à un idéal premier d'un anneau noethérien à gauche, Séminaire d'algèbre non commutative, 1971, 3.1-3.15.

[L11] L. Lesieur et R. Croisot, Extension au cas non commutatif d'un théorème de Krull et d'un lemme d'Artin-Rees, J. Reine Angew. Math. 204 (1960), 216-220. MR 24 \# A1287.

[M1] J.-M. Maranda, Injective structures, Trans. Amer. Math. Soc. 110 (1964), 98-135. MR 29 \# 1236.

[M2] E. Matlis, Injective modules over Noetherian rings, Pacific J. Math. 8 (1958), 511-528. MR 20 \# 5800. 
[M3] J. C. McConnell, Localization in enveloping rings, J. London Math. Soc. 43 (1968), 421-428. MR 37 \#4112.

[M4] G. O. Michler, Right symbolic powers and classical localization in right Noetherian rings, Math. Z. 127 (1972), 57-69.

[M5] - Example of an injective module which is not nice, Canad. Math. Bull. (to appear).

[M6] K. Morita, Localizations in categories of modules. I, Math. Z. 114 (1970), 121-144. MR 41 \#8457.

[M7] , Localization in categories of modules. II, J. Reine Angew. Math. 242 (1970), 163-169. MR 41 \#8458.

[M8] - Localization in categories of modules. III, Math. Z. 119 (1971), 313-320. MR 43 \#7462.

[M9] - Flat modules, injective modules and quotient rings, Math. Z. 120 (1971), 25-40. MR 44 \#4040.

[P1] N. Popescu, Le spectre à gauche d'un anneau, J. Algebra 18 (1971), 213-228.

[S1] W. Schelter, Two-sided rings of quotients, Arch. Math. (to appear).

[S2] W. Schelter and P. Roberts, Flat modules and torsion theories, Math. Z. (to appear).

[S3] L. Silver, Noncommutative localization and applications, J. Algebra 7 (1967), 44-76. MR. 36 \#205.

[S4] L. Small, Orders in Artinian rings. II, J. Algebra 9 (1968), 266-273. MR 37 \#6315.

[S5] B. Stenstrom, Rings and modules of quotients, Lecture Notes in Math., vol. 237, Springer-Verlag, Berlin and New York, 1971.

[W1] C. L. Walker and E. Walker, Quotient categories and rings of quotients, Rocky Mountain J. Math. 2 (1972), 513-555.

Current address: Department of Mathematics, McGill University, Montreal, Quebec, Canada 